\title{
Carbon Nanotubes Synthesis via Arc Discharge with a Yttria Catalyst
}

\author{
M. I. Mohammad, ${ }^{1}$ Ahmed A. Moosa, ${ }^{2}$ J. H. Potgieter, ${ }^{3}$ and Mustafa K. Ismael ${ }^{4}$ \\ ${ }^{1}$ Department of Chemical Engineering, University of Technology, 52 Al Sina’a Street, Baghdad, Iraq \\ ${ }^{2}$ Department of Materials, Technical College, 54 Al Zafaraniya Street, Baghdad, Iraq \\ ${ }^{3}$ School of Science and The Environment Staff, Division of Chemistry \& Environmental Science, \\ Manchester Metropolitan University, Chester Street, Manchester M1 5GD, UK \\ ${ }^{4}$ Department of Mechanical Techniques, Institute of Technology, 54 Al Zafaraniya Street, Baghdad, Iraq
}

Correspondence should be addressed to Mustafa K. Ismael; sh.on79@yahoo.com

Received 25 June 2013; Accepted 14 July 2013

Academic Editors: Y. Dai and X.-L. Dong

Copyright ( 2013 M. I. Mohammad et al. This is an open access article distributed under the Creative Commons Attribution License, which permits unrestricted use, distribution, and reproduction in any medium, provided the original work is properly cited.

\begin{abstract}
A facile method is proposed to use a computer controlled Arc discharge gap between graphite electrodes together with an yttrianickel catalyst to synthesize carbon nanotubes under an $\mathrm{Ar}-\mathrm{H}_{2}$ gases mixture atmosphere by applying different DC currents and pressure. This produces carbon nanotubes with decreased diameters and increased length. XRD evidence indicated a shift toward higher crystallinity nanotubes. Yields of the CNTs after purification were also enhanced.
\end{abstract}

\section{Introduction}

There has been intensive and increased research into the production of carbon nanotubes in recent years, as these novel nanoparticles with unique their properties are promising new advances and applications in a diverse number of areas, such as electronics, reinforced composite functionalized materials, and biomedical applications to name but a few. There are typically three major routes for such synthesis of carbon nanotubes: arc discharge, laser ablation, and chemical vapor deposition. The arc discharge technique involves an easy setup, and it is possible to obtain high yields [1]. This was the method originally used by Iijima [2] to produce multiwalled carbon nanotubes. The arc discharge is a very simple technique and is capable of massive production of both multiwalled CNTs and single-walled CNTs [3]. Arc discharge is a popular method for the production of SWNTs, and high quality SWNTs commonly collect around the cathode for easy harvesting after completion of the process [4]. The carbon arc technique for generating MWNTs appears very simple, but obtaining high yields of tubes can be difficult and requires careful control of the experimental conditions. In the most common laboratory scale production scheme, the direct current (DC) arc operates at a 1 to $4 \mathrm{~mm}$ wide gap between two graphite electrodes, which are typically between 6 and $12 \mathrm{~mm}$ in diameter and are vertically or horizontally installed in a water-cooled chamber filled with helium gas at subatmospheric pressure. Helium gas and DC current are important parameters to maximize the yield in the process [5]. Zhao et al. [6] used $\mathrm{H}_{2}-\mathrm{Ar}$ atmospheres comprising $60 \%$ Ar- $40 \% \mathrm{H}_{2}$ under a pressure of $266.6 \mathrm{mbar}$ in their work, while Luo et al. [7] and Sun et al. [8] utilised high purity hydrogen at a pressure 799.9 mbar to optimize the yield in the process. Farhat et al. [9], Hai-yan et al. [10], and Sugai et al. [11] all successfully employed an Ar atmosphere to optimize the yield of the nanotubes produced. The position of the electrode axis does not noticeably affect the MWNT quality or quantity. The $50-250 \mathrm{~mm}$ long positive electrode (anode) is consumed in the arc, while a deposit accumulates and grows on the cylindrically shaped cathode which acts as the negative electrode [5]. The carbon nanotube formation depends on the plasma density, electric field, and plasma sheath thickness. As is often the case with chemical reactions, a catalyst can significantly influence the yield of the process, and the use of molten metals or mixtures thereof is known to improve the amount of nanotubes that can be obtained in a production 


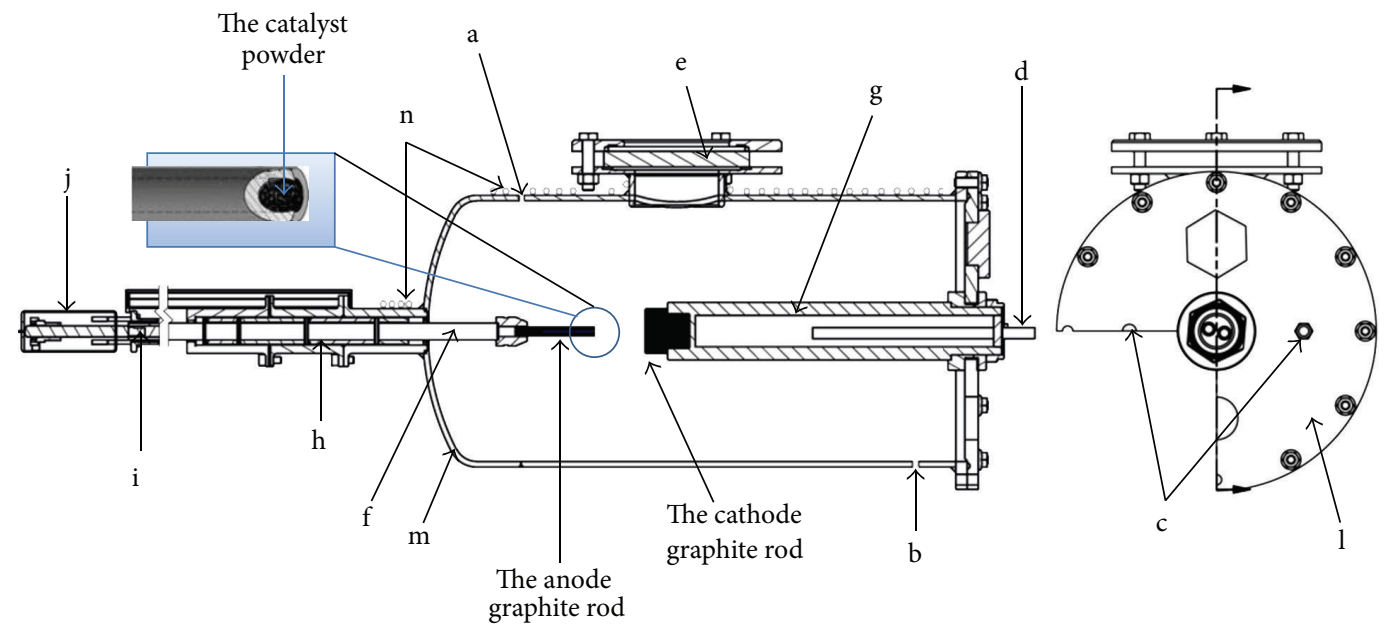

FIGURE 1: Schematic diagram of arc discharge apparatus used in present experiment: (a) a vacuum outlet, (b) gas inlet, (c) indicators of pressure and vacuum gages, (d) cathode inlet and outlet coolant water, (e) sealed quartz window, (f) high purity copper shaft coating with nickel layer, (g) high purity copper tube isolated from the chamber, (h) feed through, (i) lead screw, (j) steeper motor, (l) sealed flange cover, (m) stainless steel chamber, and (n) copper pipe for water coolant.

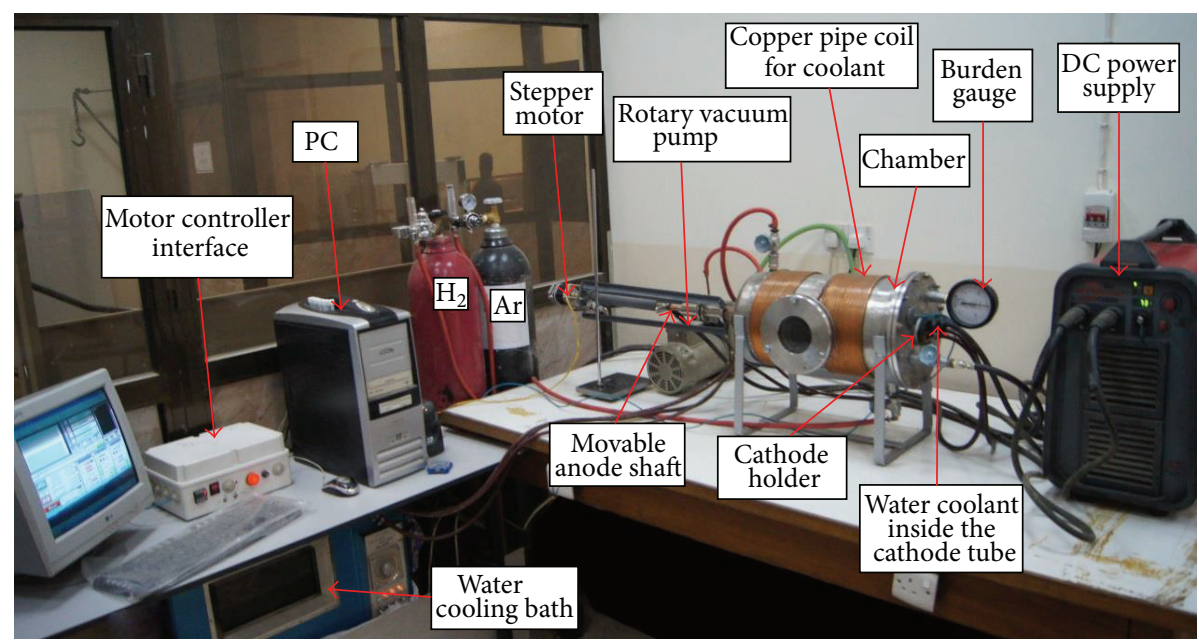

FIGURE 2: Photograph of arc discharge system setup at University of Technology, Production Engineering and Metallurgy Department.

run [12]. Growing carbon nanotubes on molten metal catalyst particles within a plasma sheath causes the carbon adatoms to diffuse to the catalyst, which can typically be incorporated into the tube structure. The diameter of the carbon nanotubes depends on the size/amount of the molten catalyst [12]. A large amount of nanotubes can, for example, be obtained by using $\mathrm{C}: \mathrm{Y}: \mathrm{Ni}$ in a $13: 1: 4$ at.\% ratio as a catalyst $[13,14]$. Dillon et al. described work using a graphite target doped with 0.6 at.\% Co and 0.6 at.\% Ni catalyst. The process occurs in a 666.6 mbar Ar environment [15]. Borisenko et al. [16] refer to a pure metal $(\mathrm{Fe}, \mathrm{Ni}$, and $\mathrm{Co})$ mixed with graphite powder as a catalyst to give a dopant/anode concentration of $0.5-3 \%$. It was also found that a maximum concentration of $\mathrm{Y}: \mathrm{Ni}=15 \%$ improved long bundle carbon nanotube yield, while a high portion of yttrium leads to the formation of sea-urchin-like structures [17]. It is clear from the literature cited previously, that a catalyst can influence both the yield and morphology of the carbon nanotubes produced during the arc process. This paper will describe further work in this area and particularly focus on the improvement in yield that can be obtained with an yttrium oxide/nickel/carbon catalyst configuration. To the best of our knowledge no previous reports could be found in the literature employing a mixture of lanthanum oxide/metal as a catalyst to increase the carbon nanotube yield in the popular carbon arc process.

\section{Experimental}

2.1. DC Arc Discharge Apparatus. Figure 1 is a schematic diagram of the DC arc discharge apparatus used in the present work. The outer diameter and length of the apparatus are $243 \mathrm{~mm}$ and $472 \mathrm{~mm}$, respectively. The chamber wall thickness and length of the apparatus are $0.3 \mathrm{~cm}$ and $122.5 \mathrm{~cm}$, respectively, and are made from 304 stainless steel. The 


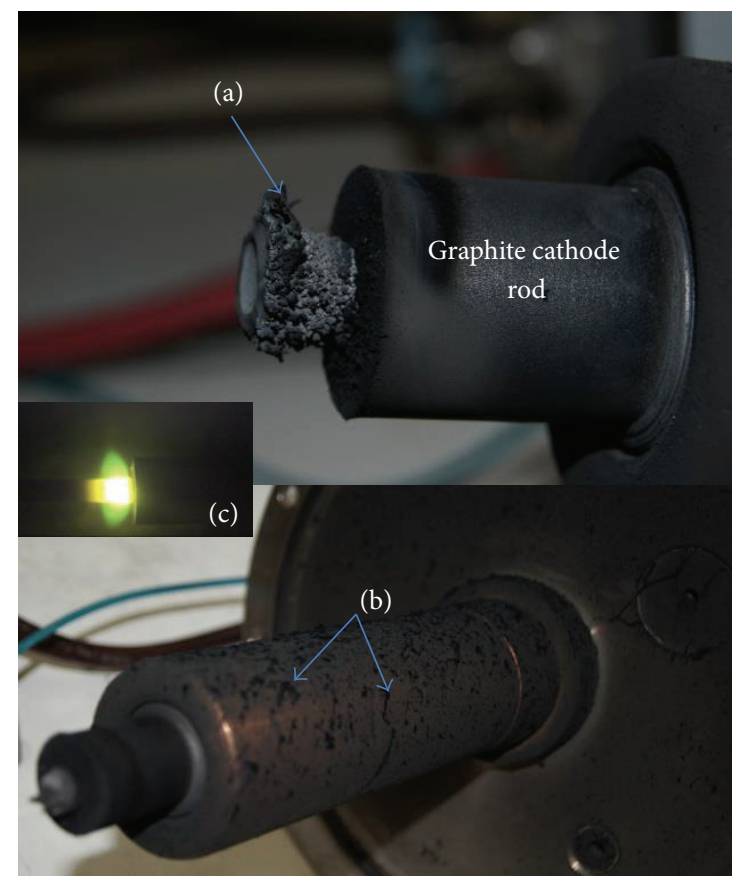

Figure 3: A photograph shows glow arc discharge: (a) deposition of soot on top surface of cathode in arc discharge apparatus; (b) fragments of web-like soot deposit on the cathode of copper cold tube holder; (c) the glow arc discharge on the left is the anode and on the right side is the cathode.

chamber is surrounded by a copper pipe coil ( $\varnothing 0.25 \mathrm{inch})$ with a length of 28 meter. The cathode tube is also cooled by a water bath and circulation from an electric pump to avoid a high temperature gradient. The design of the chamber allowed easy control of the gap distance between the two electrodes by a stepper motor (model NEMA23) for one axis, which is controlled by a NC program utilising Windows XP as a platform. The high accuracy program makes it possible to compensate for the backlash of the lead screw and thus enables one to regulate the accuracy of the gap to micrometer tolerances. This design is completely different from others reported and used for increasing both working time and yield. A rotary vacuum pump was used to evacuate the chamber to about -1000 mbar. High purity argon and hydrogen gas was then introduced at $200 \mathrm{mbar}, 0 \mathrm{mbar}, 100 \mathrm{mbar},-200 \mathrm{mbar}$, and -100 mbar. The graphite electrodes used in this investigation are made from high purity graphite (see composition in Table 1). It was cut and machined by a lath machine, producing the anode with diameter and length of $8 \mathrm{~mm}$ and $80 \mathrm{~mm}$, respectively, and a cathode with diameter and length of $25 \mathrm{~mm}$ and $20 \mathrm{~mm}$, respectively. The DC current was supplied by a high current power supply, type Cebora model 4040/T-Cell. The arc discharge generated was visible through a sealed quartz window. The layout is illustrated in Figure 1.

2.2. CNTs Synthesis. The synthesis of CNTs was performed in the DC arc discharge (Figure 2). In a typical experiment, a mixture of $1 \% \mathrm{Y}_{2} \mathrm{O}_{3}, 4 \% \mathrm{Ni}$, and $95 \% \mathrm{C}$ wt. was used as
TABLE 1

\begin{tabular}{lcccccc}
\hline Elements & $\mathrm{Al} \%$ & $\mathrm{Na} \%$ & $\mathrm{Mg} \%$ & $\mathrm{Si} \%$ & $\mathrm{~V} \%$ & $\mathrm{Fe} \%$ \\
Chemical comp. & 1.9 & $<0.035$ & 0.033 & 0.024 & 0.001 & 0.001 \\
\hline Elements & $\mathrm{S} \%$ & $\mathrm{Y} \%$ & $\mathrm{Mo} \%$ & $\mathrm{Te} \%$ & $\mathrm{Ni} \%$ & $\mathrm{C} \%$ \\
Chemical comp. & 0.015 & 0.001 & 0.001 & 0.001 & 0.0001 & $\mathrm{Bal}$. \\
\hline
\end{tabular}

the catalyst. The average grain sizes were $12 \mu \mathrm{m}, 7.5 \mu \mathrm{m}$, and $20 \mu \mathrm{m}$, respectively. The catalyst mixture was mixed with an alumina ball mill for 24 hours and then placed into the anode graphite hole (drilled $5 \mathrm{~mm}$ in diameter and $60 \mathrm{~mm}$ deep) and compacted by hand. After mounting the electrodes in the chamber, the gate was sealed and evacuation of the chamber with the rotary pump commenced until a vacuum of $-1000 \mathrm{mbar}$ was reached. The chamber was then filled with argon gas and reevacuated several times. Finally, Ar (100 mbar) was introduced into the chamber to serve as buffer gas. A stabilized arc discharge was formed by adjustment of the anode with the aid of the stepper motor NC program. The gap was measured before starting the power supply, by letting the two electrodes touch each other and setting the reference point before reversing it and, with the power on, igniting it across an approximately $\sim 0.2 \mathrm{~mm}$ gap. The resulting plasma glow could be seen through the quartz window. The arc is stopped by switching off the power supply. The anode rod is continuously consumed and deposited on the top surface of the cathode electrode. It also filled the floor of the chamber, some of the soot deposited around the chamber (cloth-like) and also on the copper cooled cathode holder. The small hard deposit collected was putted in a sample tube and investigated in a SEM, operated at $15 \mathrm{kV}$ (model: S-4160, Hitachi, Japan). XRD scans were collected on a Philips XRD with $\mathrm{Cu}-\mathrm{K} \alpha$ radiation at a wavelength of $(\lambda)=0.154 \mathrm{~nm} 2 \theta\left(20^{\circ}-60^{\circ}\right)$ and a scanning speed of 5 degree/min. Wavelength Dispersive $\mathrm{X}$-Ray Fluorescence analysis (WDXRF) was performed on a Siemens model SRS 200 to determine the chemical composition of the graphite electrodes.

\section{Results and Discussion}

Figure 3(a) shows the soot deposition on the top surface of the cathode in the arc discharge apparatus after 5 mins of starting the arc. This formation is due to the anode consumption, as can be clearly deduced from the gradual grayish deposit on the cathode surface that it can be observe by naked eye. Three types of deposition were distinguished, that is, that formed in the center of the cathode, with a multilayer deposit on the inner of the CNTs or thin light layer deposit and a thin layer cloth like deposit not too far from the center of cathode surface. A web-like soot deposited on the cold copper tube holder of the cathode is shown in Figure 3(b). SEM field emission analysis revealed straight CNTs needles in the formed range of about 10-14 $\mathrm{nm}$.

The CNT samples were investigated by Field Emission Scanning Electron Microscope (FE-SEM). The typical images are shown in Figure 4. The tubes where produced during DC arc-discharge without catalyst. Figures 4(a) and 4(b) show a carbonaceous network with needles tending to form bundles, 


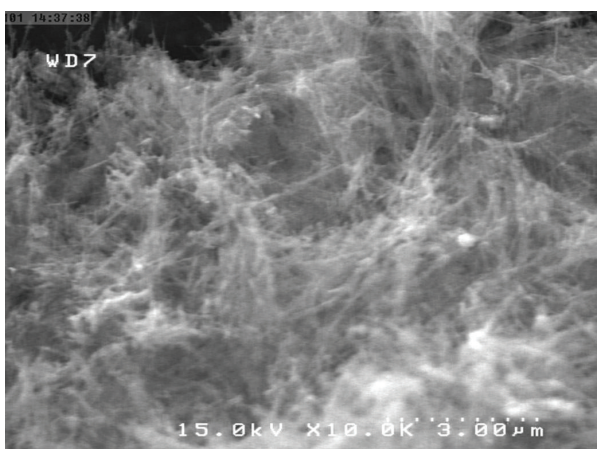

(a)

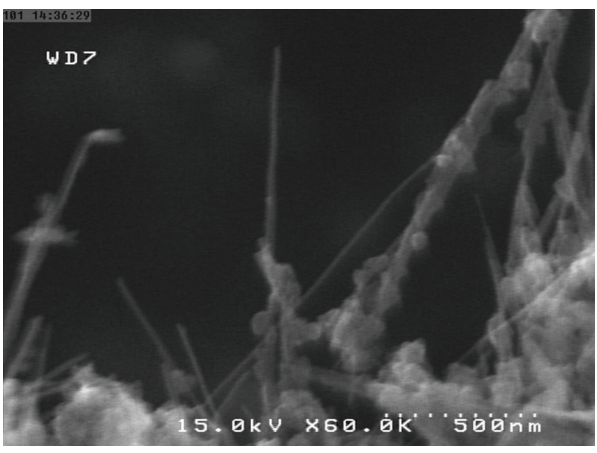

(c)

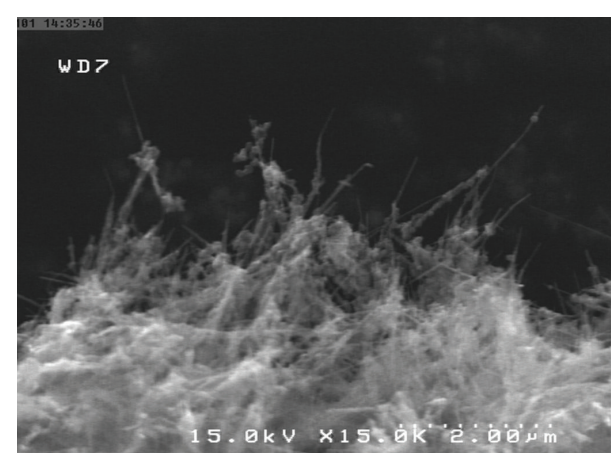

(b)

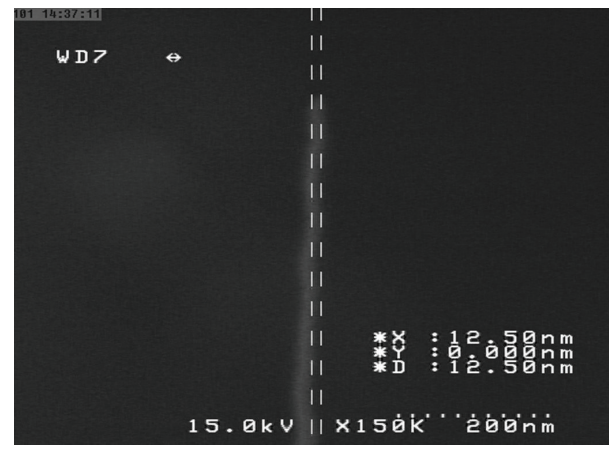

(d)

FIGURE 4: SEM micrographs for the CNTs synthesized without catalyst. ((a), (b)) Low magnification of the carbonaceous content showing needle-like CNTs. ((c), (d)) High magnification shows the diameter of tube with carbonaceous residue attached on its surface.

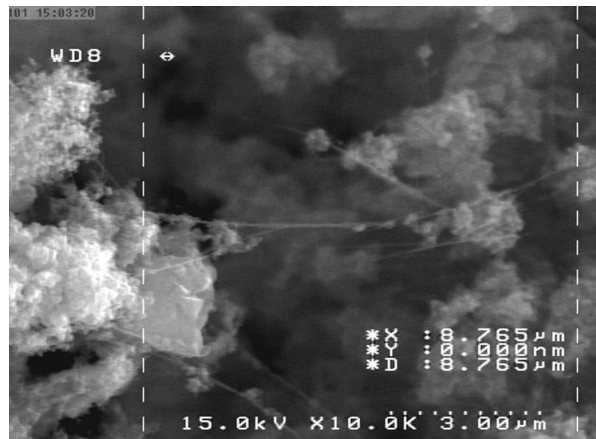

(a)

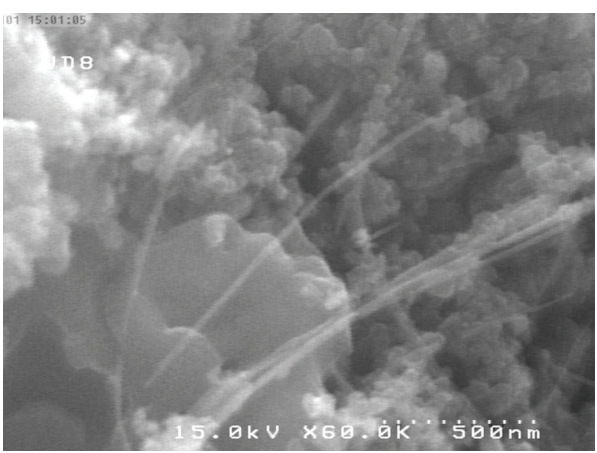

(c)

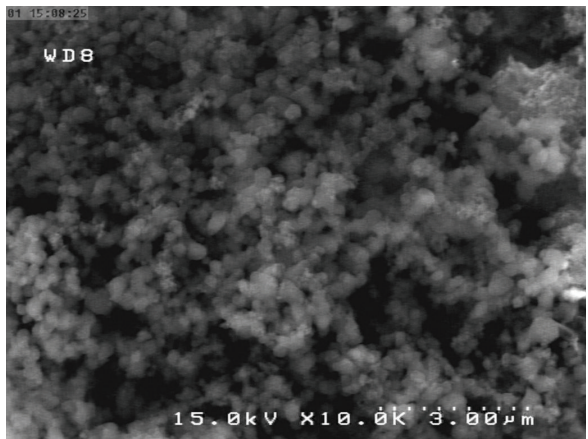

(b)

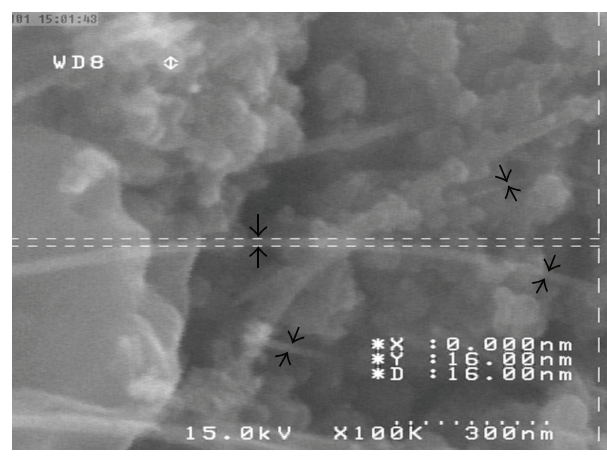

(d)

FIGURE 5: SEM micrographs for the CNTs synthesized by using $\left(\mathrm{Y}_{2} \mathrm{O}_{3}, 4 \mathrm{Ni}\right.$, and $\left.95 \mathrm{C}\right) \%$ wt. catalyst. (a) A single or bundle tubs have average length 5-8 micrometer. (b) Low magnification shows some carbonaceous with catalyst residues. ((c), (d)) High magnifications indicates free tubes surface defects with average tube diameter $\sim 10 \mathrm{~nm}$. 


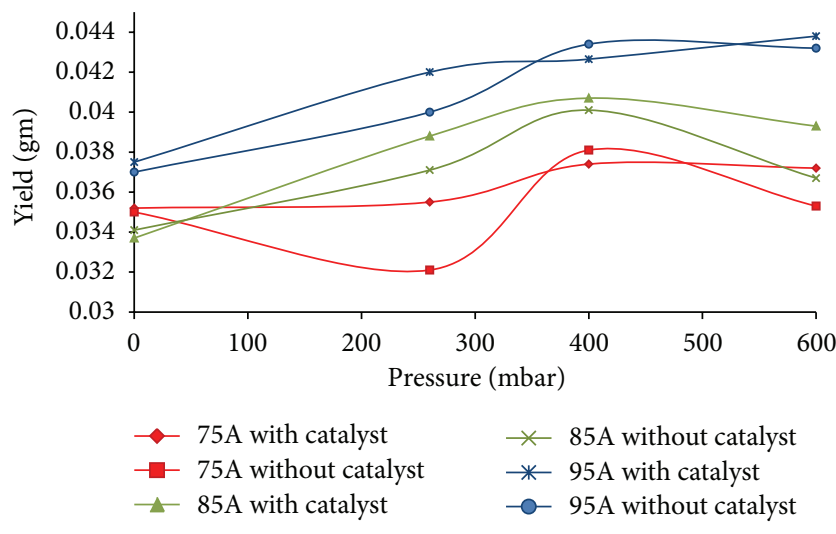

FIGURE 6: The plot of yield versus the amount of pressure of $\left(1 \mathrm{Y}_{2} \mathrm{O}_{3}\right.$, $4 \mathrm{Ni}$, and $95 \mathrm{C}) \% \mathrm{wt}$. with and without catalyst for 5 mins of graphite anode ablation using $60 \% \mathrm{Ar}-40 \% \mathrm{H}_{2}$ mixture gases.

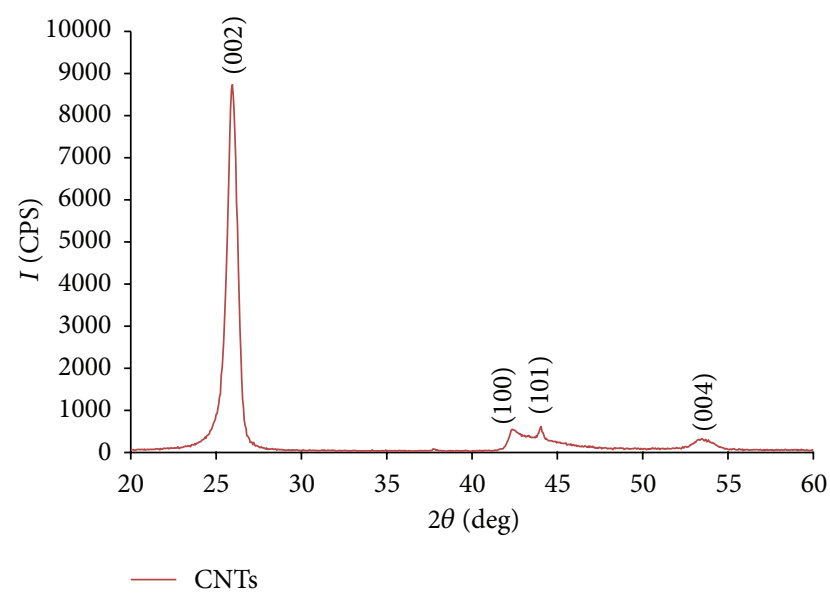

FIGURE 7: XRD pattern of CNTs synthesized by Arc discharge.

which have relatively large tube diameters if compared to the tubes produced when using a $\mathrm{Y}_{2} \mathrm{O}_{3} / \mathrm{Ni} / \mathrm{C}$ catalyst as shown in Figures 5(a) and 5(b). Further high magnifications shown in Figures 5(c) and 5(d) indicate that a nanoparticle formed and is present due to the catalyst used, and that everything aggregates as a result of van der Waals interactions. When comparing the length: diameter ratio $(L / D)$ of $\sim 120$ of the nanotubes produced without catalyst in Figure 4 to those of the carbon nanotubes produces with the catalyst of $\sim 484$ as shown in Figure 5, it is obvious that the catalyst increases the tube lengths. Furthermore, tubes produced with the catalyst have an even diameter and can occur as SWNTs, while tubes produced without the catalyst tend to have uneven diameters and mostly occur as MWNTs with structural defects such as kinks and incompletely formed walls. The catalyst seems to induce structured carbon atom build-up on the nanoparticles of the catalysts during the plasma process, while in the absence of the catalyst random build-up of the carbon atoms on themselves occur.

The purification treatment consisted of $0.5 \mathrm{~g}$ of soot sonicated with $12 \mathrm{M} \mathrm{HCl}(200 \mathrm{~mL})$ for $30 \mathrm{mins}$ and then soaking

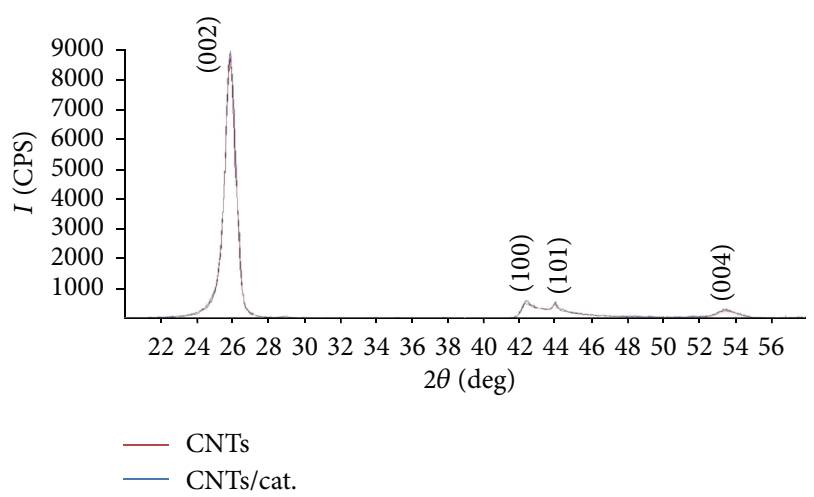

FIGURE 8: XRD pattern of CNTs synthesized by Arc discharge with and without catalyst compared with graphite pattern.

it overnight. In the next step the soot was refluxed in $6 \mathrm{M}$ of $\mathrm{HCl}(200 \mathrm{~mL})$ for 6 hours, followed by a second reflux step with $2.8 \mathrm{~N} \mathrm{HNO}_{3}(200 \mathrm{~mL})$ also for 6 hours, before being filtered and washed 5 times with distilled water until the $\mathrm{pH}$ reached a value of 7 . Thereafter the product was dried in an drying oven at $100^{\circ} \mathrm{C}$ for 1 hour, then the product followed by oxidation treatments, this process occurred at $550^{\circ} \mathrm{C}$ for 30 mins. The yields after purification treatments when using different pressures of high purity $60 \% \mathrm{Ar}-40 \% \mathrm{H}_{2}$ gases mixture for 5 mins of arcing time are shown in Figure 6. It is clear from the curves in Figure 6 that there is a noticeable difference in yield between approximately 100 to 350 mbar of gas mixture employed when using the catalyst in the CNT preparations, regardless of the current used for the arc. As the arc current increases, so does the yield. The maximum difference between the yields with and without catalyst is observed around $250 \mathrm{mbar}$ gas pressure for all the different current densities used. A good yielding range for all the production runs seems to be between approximately 250 and 400 mbar gas pressure.

$\mathrm{XRD}$ characterization is normally used to obtain some information on interlayer spacing, the structure strain and impurities [18], and average wall numbers [19] in CNTs. The main features of the typical X-ray diffraction pattern of CNTs are close to those of graphite. The X-ray diffraction profile is useful to differentiate microstructural details between the CNTs and graphite and can help to determinate the sample purity (e.g., catalyst, functional groups) [18]. The XRD pattern of pure graphite displays a sharp characteristic peak at $26.34^{\circ} 2 \theta$, which corresponds to the (002) diffracted plane, with further small peaks at $42.61^{\circ}, 44.831^{\circ}$, and $54.231^{\circ}$ [20]. The graphite interlayer spacing of the (002) plane is $0.33 \mathrm{~nm}$. The XRD pattern of the CNTs grown by the arc-discharge in this investigation is shown in Figure 7. The main peaks correspond to the 002 plane with $2 \theta$ equal to $25.97^{\circ}$. Thus, it was found that the (002) diffraction shifts from $26.34^{\circ}$ to $25.97^{\circ}$ for these carbon nanotubes, which corresponds to a spacing of $0.34 \mathrm{~nm}$. For MWNTs, the shape, width, and intensity of the (002) diffraction peak are modified with the increase in the diameter of the nanotube [21]. It can estimate number of 
average walls from the contribution of the intertube (outerwall contacts) structure with the intratube structure (concentric shells) regarding the peak (002) because the contribution of intratube increased linearly with walls numbers, while the contribution does not increased which appears slightly asymmetric towards lower angles. The asymmetry is due to the presence of different crystalline species [22]. The XRD patterns of MWNTs produced with and without catalyst compared with graphite are presented in Figure 8.

The characteristic peak of MWNTs is around $25.97^{\circ}$ for MWNTs with and without catalyst. This is in agreement with similar work reported earlier [23]. The detected pattern can be assigned to the CNTs crystal planes of (002), while (100) and (101) could also be assigned to graphene [24]. However, compared to the normal graphite XRD pattern, the strongest CNT peak (002) is broad and weakened and shows a slight downward shift to $2 \theta=25.9^{\circ}$ (for graphite, $2 \theta$ equals $26.34^{\circ}$ ). The broadening indicates that the long range order in the CNT is lower than that in graphite. In addition, the $2 \theta$ downward shift for the CNTs relates to an increase of the $\mathrm{sp}^{2} \mathrm{C}=\mathrm{C}$ layers spacing [25]. The peak for the MWNT becomes broad and weakened, which can be attributed to the high curvature and high strain energy resulting from the small diameter of the MWNTs. Other peaks at $42.4^{\circ}$ and $43.98^{\circ}$ are also observed, and correspond to the $\{(100),(100)\}$, crystallography planes [22].

\section{Conclusions}

The use of a $\left(1 \mathrm{Y}_{2} \mathrm{O}_{3}, 4 \mathrm{Ni}\right.$, and $\left.95 \mathrm{C}\right) \%$ wt. catalyst had a significant effect on the yields of CNTs produced by using the arc discharge method under an atmosphere of high purity $60 \%$ Ar- $40 \% \mathrm{H}_{2}$ gases mixture. Field emission SEM reveals a small decrease in the tube diameters and an increase in length gradually. The yield of the CNTs increased significantly with using a catalyst at 250 to 400 mbar. XRD scans show a shifting of the main peaks towards lower values, which is an indication of the increase in the $\mathrm{sp}^{2} \mathrm{C}=\mathrm{C}$ layers spacing. Thus it is clear that yttrium oxide/nickel increases both yield and length of tubes and further decreases the tubes diameter.

\section{Acknowledgments}

The authors would like to thank all those who have helped make this work possible, that is, the University of Technology, Baghdad, which supplied the high temperature lab. Also one of the authors (Mustafa K. Ismael) thanks the Ministry of Science and Technology/Department of Chemistry Research and Materials Physics/Applied Physics Research Centre, Baghdad, for supplying the chemical compositions of the graphite rods. Thanks are also due to the Iraq Geological Survey for support with the XRD test.

\section{References}

[1] R. Duncan, V. Stolojan, and C. Lekakou, "Manufacture of carbon multi-walled nanotubes by the arc discharge technique," in Proceedings of the World Congress on Engineering, 2007.

[2] S. Iijima, "Helical microtubules of graphitic carbon," Nature, vol. 354, no. 6348, pp. 56-58, 1991.
[3] Y. S. Park, K. S. Kim, H. J. Jeong et al., "Low pressure synthesis of single-walled carbon nanotubes by arc discharge," Synthetic Metals, vol. 126, no. 2-3, pp. 245-251, 2002.

[4] C. Journet, W. K. Maser, P. Bernier et al., "Large-scale production of single-walled carbon nanotubes by the electric-arc technique," Nature, vol. 388, no. 6644, pp. 756-758, 1997.

[5] M. Meyyappan, Carbon Nanotubes Science and Applications, CRC Press, 2005.

[6] X. Zhao, S. Inoue, M. Jinno, T. Suzuki, and Y. Ando, "Macroscopic oriented web of single-wall carbon nanotubes," Chemical Physics Letters, vol. 373, no. 3-4, pp. 266-271, 2003.

[7] S. Luo, D. Huang, Y. Huang, X. Dou, and X. Zhao, "Orderly evolution in the morphology of the anode deposit in hydrogen arc discharge," Carbon, vol. 43, no. 1, pp. 109-115, 2005.

[8] X. Sun, W. Bao, Y. Lv, J. Deng, and X. Wang, "Synthesis of high quality single-walled carbon nanotubes by arc discharge method in large scale," Materials Letters, vol. 61, no. 18, pp. 39563958, 2007.

[9] S. Farhat, M. L. De La Chapelle, A. Loiseau et al., "Diameter control of single-walled carbon nanotubes using argon-helium mixture gases," Journal of Chemical Physics, vol. 115, no. 14, pp. 6752-6759, 2001.

[10] Z. Hai-yan, C. Jian, L. Song-hao et al., "The Raman scattering of carbon nanotubes produced in different inert gases and their pressures by arc discharge," Chinese Physics, vol. 9, no. 5, pp. 375-378, 2000.

[11] T. Sugai, H. Omote, S. Bandow, N. Tanaka, and H. Shinohara, "Production of fullerenes and single-wall carbon nanotubes by high-temperature pulsed arc discharge," Journal of Chemical Physics, vol. 112, no. 13, pp. 6000-6005, 2000.

[12] M. Keidar, I. Levchenko, T. Arbel, M. Alexander, A. M. Waas, and K. K. Ostrikov, "Magnetic-field-enhanced synthesis of single-wall carbon nanotubes in arc discharge," Journal of Applied Physics, vol. 103, no. 9, Article ID 094318, 2008.

[13] J. Qiu, Y. Li, Y. Wang et al., "High-purity single-wall carbon nanotubes synthesized from coal by arc discharge," Carbon, vol. 41, no. 11, pp. 2170-2173, 2003.

[14] A. Hassanien, M. Tokumoto, Y. Kumazawa et al., "Atomic structure and electronic properties of single-wall carbon nanotubes probed by scanning tunneling microscope at room temperature," Applied Physics Letters, vol. 73, no. 26, pp. 3839-3841, 1998.

[15] A. Dillon, T. Gennett, K. M. Jones, J. L. Alleman, P. A. Parilla, and M. J. Heben, "A simple and complete purification of singlewalled carbon nanotubes materials," Advanced Materials, vol. 11, no. 16, 1999.

[16] D. N. Borisenko, N. N. Kolesnikov, M. P. Kulakov, and V. V. Kveder, "Growth of Carbon Nano-Tubes (CNT) in electric-arc discharge in argon," International Journal of Nanoscience, vol. 1, no. 3/4, pp. 235-246, 2002.

[17] J. Gavillet, A. Loiseau, O. Stephan, S. Tahir, and P. Bernier, "Investigation of the role of Yttrium in the production of SWNT by arc discharge," AIP Conference Proceedings, vol. 544, pp. 222225, 2000.

[18] T. Belin and F. Epron, "Characterization methods of carbon nanotubes: a review," Materials Science and Engineering B, vol. 119, no. 2, pp. 105-118, 2005.

[19] D. N. Futaba, T. Yamada, K. Kobashi, M. Yumura, and K. Hata, "Macroscopic wall number analysis of single-walled, doublewalled, and few-walled carbon nanotubes by X-ray diffraction," Journal of the American Chemical Society, vol. 133, no. 15, pp. 5716-5719, 2011. 
[20] The International Centre for Diffraction Data (ICDD), X-Ray powder diffraction data (PDF), Number (00-001-0640), http:// www.icdd.com/.

[21] E. Boccaleri, A. Arrais, A. Frache, W. Gianelli, P. Fino, and G. Camino, "Comprehensive spectral and instrumental approaches for the easy monitoring of features and purity of different carbon nanostructures for nanocomposite applications," Materials Science and Engineering B, vol. 131, no. 1-3, pp. 72-82, 2006.

[22] S. C. Tjong, Carbon Nanotube Reinforced Composites, WILEYVCH, Weinheim, Germany, 2006.

[23] C. Liu, H. Li, and S. Fan, "Homogeneous carbon nanotube/carbon composites prepared by catalyzed carbonization approach at low temperature," Journal of Nanomaterials, vol. 2011, Article ID 281490, 5 pages, 2011.

[24] X. P. Gao, Y. Zhang, X. Chen et al., "Carbon nanotubes filled with metallic nanowires," Carbon, vol. 42, no. 1, pp. 47-52, 2004.

[25] H.-B. Zhang, G.-D. Lin, Z.-H. Zhou, X. Dong, and T. Chen, "Raman spectra of MWCNTs and MWCNT-based $\mathrm{H}_{2}$-adsorbing system," Carbon, vol. 40, no. 13, pp. 2429-2436, 2002. 

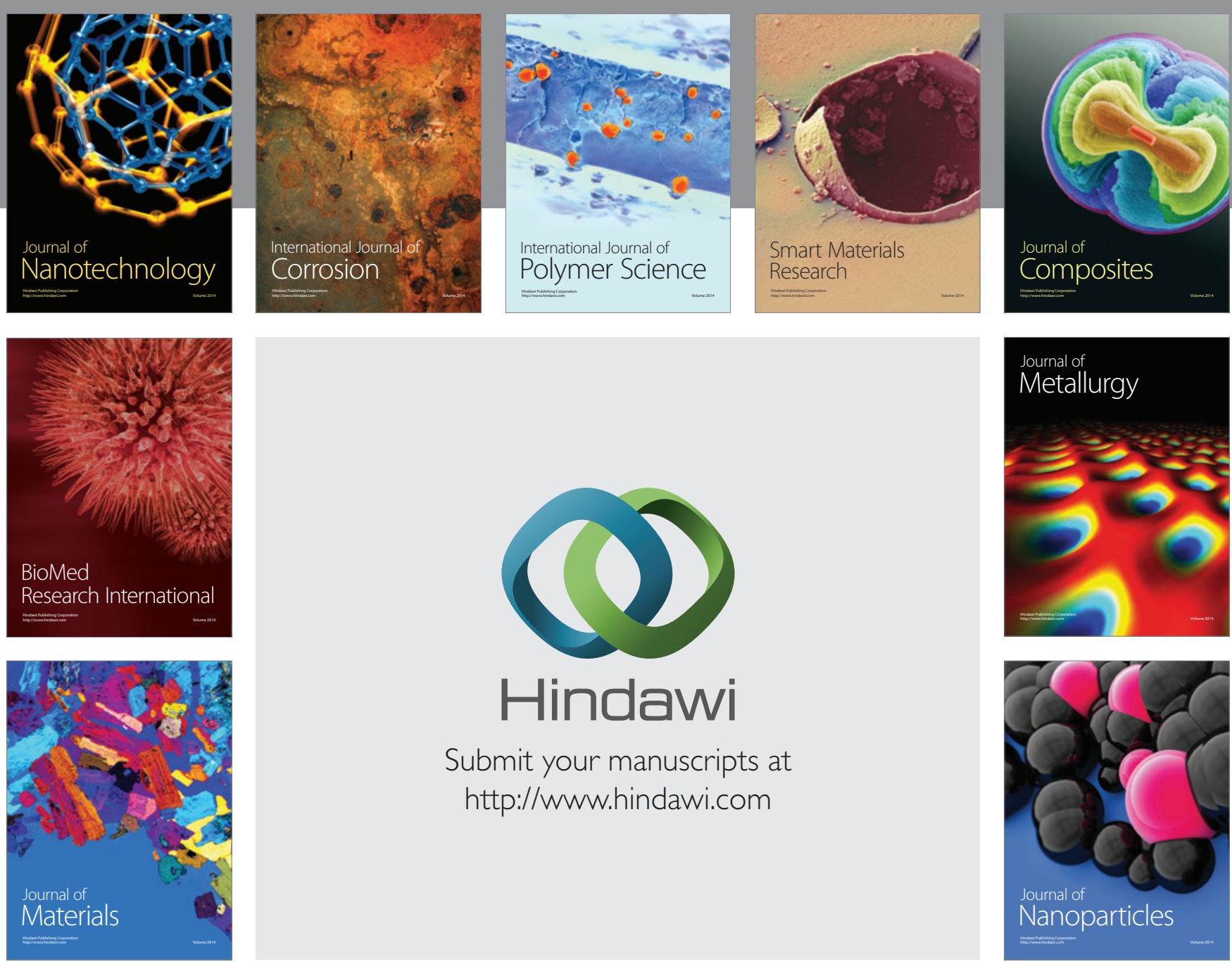

Submit your manuscripts at http://www.hindawi.com
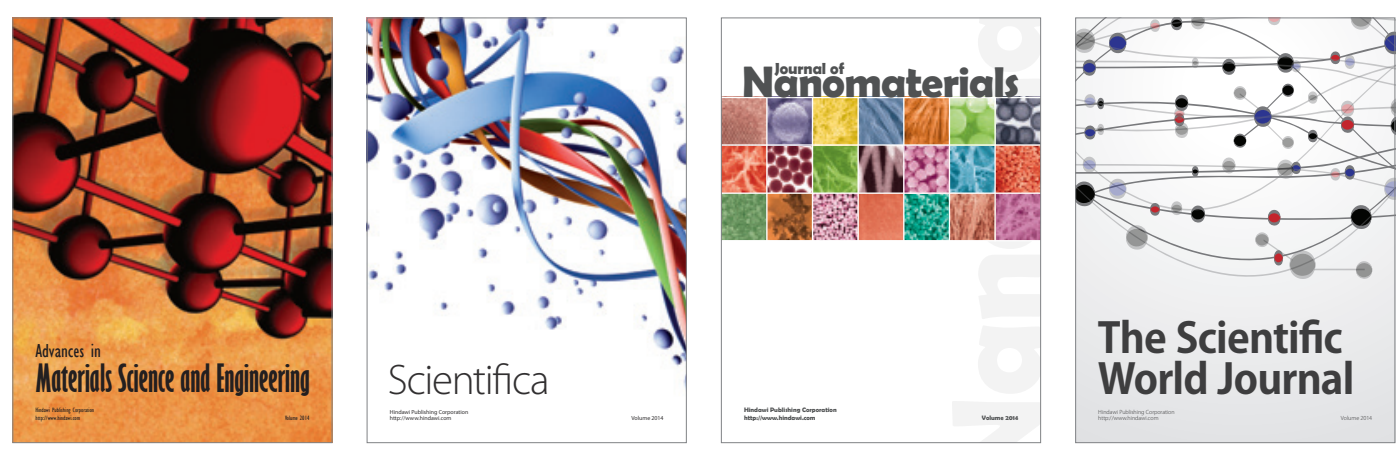

\section{The Scientific World Journal}
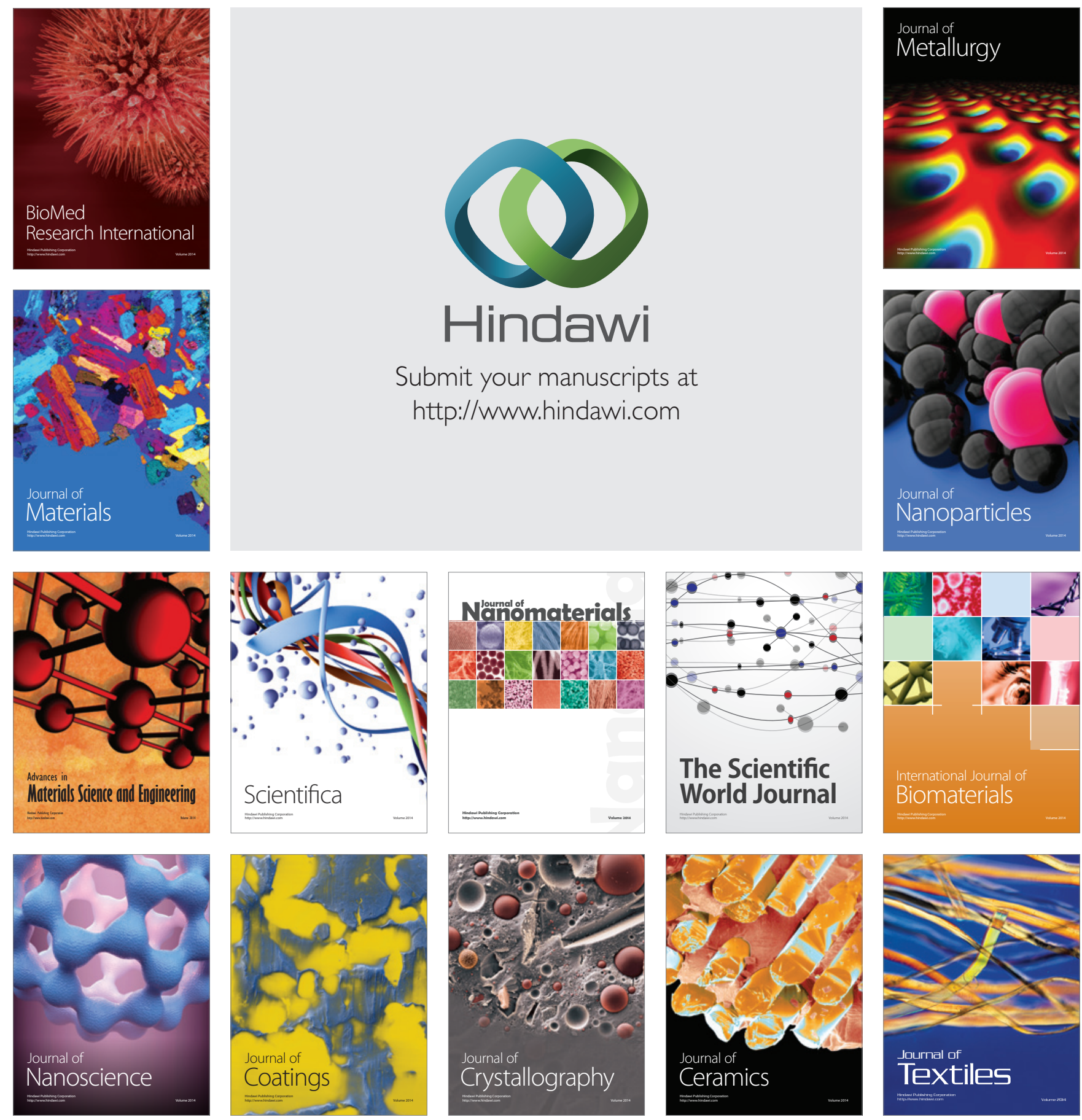